\title{
Propagation of Pore Pressure and Stress in Saturated Porous Media Based on a Darcy-Brinkman Formulation
}

\author{
Duoxing Yang $\mathbb{D}^{1}$ and Lianzhong Zhang ${ }^{2}$ \\ ${ }^{1}$ National Institute of Natural Hazards, Ministry of Emergency Management of China, 100085 Beijing, China \\ ${ }^{2}$ School of Physics, Nankai University, 300071 Tianjin, China \\ Correspondence should be addressed to Duoxing Yang; dxyangcea@yahoo.com
}

Received 5 August 2021; Revised 18 October 2021; Accepted 3 December 2021; Published 27 December 2021

Academic Editor: Yingfang Zhou

Copyright (c) 2021 Duoxing Yang and Lianzhong Zhang. This is an open access article distributed under the Creative Commons Attribution License, which permits unrestricted use, distribution, and reproduction in any medium, provided the original work is properly cited.

\begin{abstract}
Propagation of pore pressure and stress in water-saturated elastic porous media is theoretically investigated when considering the Darcy-Brinkman law. The wave mode, phase velocity, phase lag, damping factor, and characteristic frequency are found from the updated mathematic model. The Brinkman term describes the fluid viscous shear effects and importantly contributes to the dispersion relation and wave damping. The coincidence of the properties of Biot waves of the first and second kinds occurs at a characteristic frequency, which is remarkably influenced by the Brinkman term. A key finding is that, compared to the Darcy-Brinkman law, Darcy's law overestimates the phase velocity, damping, and phase lag of the first wave, while underestimates the phase velocity, damping, and phase difference of the second wave. The introduction of the Darcy-Brinkman law yields an improved description of the damping of the compressional wave modes in saturated porous media.
\end{abstract}

\section{Introduction}

Propagation of acoustic waves through saturated porous media is a phenomenon of great fundamental importance and increasing technological relevance [1], which is significant to obtain better surface acoustic wave device's performance. Compressional waves in saturated porous media have found geophysical interests to hydraulic fracturing, oil exploration, volcanic eruptions, and magneto-electro-elastic behavior of piezoelectric nanostructures [2]. Different investigations are available in literature focusing on the mechanical behaviors of piezoelectric nanostructures $[3,4]$ on fields of linear and nonlinear, longitudinal and transverse, free and forced wave propagation in microscale. Initial and couple stress influence on transmission of the Love-type wave [5], and Rayleigh waves [6] in material layers with imperfect interface [7], and surface wave propagation between viscous liquid and initially stressed piezoelectric half-space [8] are investigated by using the spatial varying WKB (WentzelKramers-Brillouin) method $[9,10]$. Solutions are obtained by a perturbation technique in closed-form for normal, shear stresses, dielectric displacement, and electric potential [11], and it is found that the complex phase velocity with positive imaginary part increases with time [12].

As is well known, the first and second kind of compressional waves exist in saturated porous media, which are predicted by the Biot theory $[13,14]$. The wave mode, dispersion relation $[15,16]$, and transfer function [17] are proposed. Generally, the first kind of compressional wave, which is known as Biot's wave of the first kind or the first wave, is a weakly decaying high-velocity mode, while the second kind of compressional wave, which is known as Biot's wave of the second kind or the second wave, is a strongly damping slow-velocity mode [18-21]. The nonlinear waves are characterized with diffusive, oscillatory, and shock types in the two-phase fluid saturated porous media [22-24]. Strong viscous coupling causes the coalescence of the two wave fronts into a single front, and the saturated porous medium behaves a single continuum exhibiting internal dissipation [16]. The coincidence of the properties of Biot waves of the first and second kinds occurs at a characteristic frequency, at which the properties of the first and 
second waves coincide [25]. Under conditions of two-phase flows, the resonance frequency of the gas is the governing factor for the transition of the wave modes [26]. The strong decay of compressional waves is attributed to the viscous coupling effect $[27,28]$ and the Darcy viscous resistance [29-31]. Elastic waves in non-Newtonian (Maxwell) fluidsaturated porous media are deeply investigated [32, 33], and it is found that the introduction of a Maxwell fluid into the Biot theory results in an overall increase of the attenuation of three Biot waves in the intermediate frequency domain except in the deeply non-Newtonian regime.

Based on the Biot theory, the interaction forces due to the relative motion between fluid and solid phases [14, 17, 34] are formulated by the linear Darcy's law, which relates macroscopic pressure gradients to the flow velocity vector in the fluid phase, describes the solid viscous drag effects [35], and hence, neglects the internal friction arising from viscous shear stress $[36,37]$. For the flow of fluids in dualporosity media (e.g., macropores and micropores) or miniature-sized porous structures, viscous shear stress is significant $[1,37,38]$. Rigorous usage of spatial averaging methods [39] results in the Darcy-Brinkman equation, which is known as Darcy's law with Brinkman correction or the Darcy-Brinkman law [35]. The Brinkman term in the Darcy-Brinkman law $[36,37,39]$ describes the fluid viscous shear effects that oppose the flow through the porous structures [36]. Besides the drag considered in Darcy's law, the Darcy-Brinkman law accounts for internal friction [37, 38] which is described by the Stokes equation at the fluidporous medium boundary, and has considerable influences on the momentum transfer between the solid and the fluid in porous media [40].

Biot [14] pointed that the assumption of Darcy's law breaks down if the frequency exceeds a certain value, known as Biot's critical frequency above which the compressional wave attenuation is controlled by the viscous shear stress and the inertial effects. However, Darcy's law does not include the internal friction and the inertia effects. Recently, a macroscopic model for describing compressional wave propagation in saturated porous media was proposed, and the Forchheimer term (e.g., inertial effects) was added to Euler's equation without the Brinkman term included [41]. A Darcy-Brinkman-Biot formulation [37] was developed, which approximates the Navier-Stokes equations in fluidfilled macropores and resembles the equations for poroelasticity in microporous regions. The Darcy-Brinkman-Biot formulation neglects inertial effects (e.g., Forchheimer term) in a momentum transfer term (drag) between the fluid and the solid phases, given that the Reynolds number within the microporous region is small. There arises a problem in which it is required to identify the effects of internal friction on the compressional wave propagation in saturated porous media. That is why the emphasis here is placed on investigation of the wave propagation and damping to the DarcyBrinkman law.

In this paper, with the help of the Darcy-Brinkman law $[35,37,39]$, the momentum conservation laws $[14,17]$ in their linearized harmonic form, which describes the onedimensional propagation of compressional waves in satu- rated porous media, are extended from the Biot theory. The dispersion relation of the wave modes is reformulated. A characteristic frequency for the coincidence of the properties of Biot waves of the first and second kinds is found. We analyze the conditions under which the properties of the two compressional waves coincide. The transfer functions for pore pressures and stresses are constructed. Comparisons between the Darcy-Brinkman law and Darcy's law in the compressional wave motion are carried out. The introduction of the Darcy-Brinkman law may yield a better understanding of the compressional waves in saturated porous media.

\section{Mathematical Formulation of the Problem}

The governing equations of the Biot theory $[14,17]$ and the Darcy-Brinkman-Biot formulation [37] in saturated porous media were established through use of the conservation laws. Sufficient detail of the mass and momentum conservation laws in time domain was provided by Biot [14] and by Carrillo and Bourg [37]. Recently, based on the Biot theory, the equations for compressional waves in frequency domain was developed by van der Grinten et al. [17]. Here, primary attention is paid to the introduction of the Brinkman term to the Biot theory in frequency domain. Suppose that caret $(\wedge)$ represents the complex amplitude, where $j^{2}=-1 . \widehat{p}$ and $\widehat{\sigma}$ are the pore pressure and effective stress. $\widehat{u}$ and $\widehat{w}$ represent the actual velocities of the fluid and solid phases. $\varphi$ and $\kappa$ are the porosity and frequency-independent permeability. $\rho_{l}$ and $\rho_{p}$ denote the intrinsic densities of the fluid and solid phases, respectively. $\omega$ is the angular frequency. $K_{l}$ and $K_{p}$ are, respectively, the bulk modulus of the fluid and the constrained modulus of the solid. The added mass coefficient $\alpha_{\infty}$ is related to $\rho_{a}$ by $\rho_{a}=\left(\alpha_{\infty}-1\right) \varphi \rho_{l}$. The critical Biot frequency [14] is given by $\omega_{c}=\eta \varphi /\left(\kappa \rho_{l}\right)$.

It is assumed that the intrinsic density of the solid is constant, and the solid elastic deformation is fully described in terms of the porosity $[14,17]$. Considering a $e^{j \omega t}$ dependence for all relevant variables, the equations governing the one-dimensional propagation of compressional waves in the linearized harmonic form [17] are the following.

$$
\begin{gathered}
\frac{j \omega}{\varphi \rho_{l}}\left(\varphi \widehat{\rho}_{l}+\rho_{l} \widehat{\varphi}\right)+\frac{\partial \widehat{w}}{\partial x}=0 \\
-j \omega \widehat{\varphi}+(1-\varphi) \frac{\partial \widehat{u}}{\partial x}=0 \\
j \omega \varphi \rho_{l} \widehat{w}=-\varphi \frac{\partial \widehat{p}}{\partial x}+\left(j \omega \rho_{a}+\frac{\varphi^{2} \eta}{\kappa}\right)(\widehat{u}-\widehat{w}) \\
j \omega(1-\varphi) \rho_{p} \widehat{u}=-\frac{\partial \widehat{\sigma}}{\partial x}-(1-\varphi) \frac{\partial \widehat{p}}{\partial x}-\left(j \omega \rho_{a}+\frac{\varphi^{2} \eta}{\kappa}\right)(\widehat{u}-\widehat{w}) .
\end{gathered}
$$

Equations (1) and (2) are the mass conservation laws for the fluid and the solid, respectively. Equations (3) and (4) represent the conservation of momentum of the fluid and 
the solid, respectively, as stated in the Biot theory [14]. The term, $\varphi^{2} \eta / \kappa(\widehat{u}-\widehat{w})$ in Equations (3) and (4) is the fluidsolid momentum transfer term $[14,37]$ and describes the frequency-dependent interaction forces between fluid and solid and is expressed as a function of the relative velocities of the two phases [37]. The high frequency added mass, $j \omega$ $\rho_{a}$ describes the purely imaginary interaction force $j \omega \rho_{a}(\widehat{u}$ $-\widehat{w})$ in the limit for high frequencies [17]. The Brinkman term [39] is defined by $\varphi \eta\left(\partial^{2}(\widehat{u}-\widehat{w}) / \partial x^{2}\right)$, which recovers the viscous drag effects and uses the fluid viscosity coefficient [36-38]. According to the momentum conservation laws of the Biot theory [14], we introduce the Brinkman term to the momentum conservation of the fluid and the solid, as stated in the reference [37]. From Equations (3) and (4), it follows that

$$
\begin{gathered}
j \omega \varphi \rho_{l} \widehat{w}=-\varphi \frac{\partial \widehat{p}}{\partial x}+\varphi \eta \frac{\partial^{2}(\widehat{u}-\widehat{w})}{\partial x^{2}}+\left(j \omega \rho_{a}+\frac{\varphi^{2} \eta}{\kappa}\right)(\widehat{u}-\widehat{w}), \\
j \omega(1-\varphi) \rho_{p} \widehat{u}=-\frac{\partial \widehat{\sigma}}{\partial x}-(1-\varphi) \frac{\partial \widehat{p}}{\partial x}-\varphi \eta \frac{\partial^{2}(\widehat{u}-\widehat{w})}{\partial x^{2}}-\left(j \omega \rho_{a}+\frac{\varphi^{2} \eta}{\kappa}\right)(\widehat{u}-\widehat{w}) .
\end{gathered}
$$

Equations (1), (2), (5), and (6) is the Darcy-BrinkmanBiot formulation in the frequency domain under the rigorous assumptions present in the Biot theory $[14,17]$. Inserting a harmonic $x$-dependence $e^{-j \omega x / c}$ for all dependent variables in equations (1), (2), (5), and (6), and following the procedure [17], the constitutive equations of pore pressure and stress are given by

$$
\begin{gathered}
\widehat{p}=K_{l}\left(\frac{\widehat{w}}{c}+\frac{1-\varphi}{\varphi} \frac{\widehat{u}}{c}\right), \\
\widehat{\sigma}=K_{p} \frac{\widehat{u}}{c} .
\end{gathered}
$$

Substituting Equations (7) and (8) into Equations (5) and (6), it yields

$$
\begin{aligned}
& \widehat{w}\left(\rho_{22}-\frac{\varphi K_{l}}{c^{2}}-\frac{j \varphi^{2} \eta}{k \omega}+\frac{j \varphi \eta \omega}{c^{2}}\right) \\
& =\widehat{u}\left(-\rho_{12}+\frac{(1-\varphi) K_{l}}{c^{2}}-\frac{j \varphi^{2} \eta}{k \omega}+\frac{j \varphi \eta \omega}{c^{2}}\right),
\end{aligned}
$$

$$
\begin{aligned}
& \widehat{w}\left(-\rho_{12}+\frac{(1-\varphi) K_{l}}{c^{2}}-\frac{j \varphi^{2} \eta}{k \omega}-\frac{j \varphi \eta \omega}{c^{2}}\right) \\
& =\widehat{u}\left(\rho_{11}-\frac{K_{p}}{c^{2}}-\frac{(1-\varphi)^{2} K_{l}}{\varphi c^{2}}-\frac{j \varphi^{2} \eta}{k \omega}-\frac{j \varphi \eta \omega}{c^{2}}\right) .
\end{aligned}
$$

By virtue of Equations (9) and (10), the dispersion relation takes the following form

$$
\chi c^{4}+\beta c^{2}+\gamma=0
$$

with

$$
\begin{aligned}
\chi= & \rho_{11} \rho_{22}-\rho_{12}^{2}-\frac{j \varphi^{2} \eta}{\kappa \omega}\left(\rho_{11}+\rho_{22}+2 \rho_{12}\right), \\
\beta= & -\rho_{22} K_{p}-\frac{(1-\varphi)^{2} K_{l} \rho_{22}}{\varphi}-\varphi K_{l} \rho_{11}+2(1-\varphi) K_{l} \rho_{12} \\
& +\frac{j \varphi \eta\left(K_{l}+\varphi K_{p}\right)}{\kappa \omega}+j \varphi \eta \omega\left(\rho_{11}-\rho_{22}\right),
\end{aligned}
$$$$
\gamma=\varphi K_{l} K_{p}+j \varphi \eta \omega\left(-K_{p}+\frac{2 \varphi-1}{\varphi} K_{l}\right)
$$

The terms $j \varphi \eta \omega\left(\rho_{11}-\rho_{22}\right)$ and $j \varphi \eta \omega\left(-K_{p}+(2 \varphi-1 / \varphi)\right.$ $\left.K_{l}\right)$ in the coefficients of $\beta$ and $\gamma$ implies the contribution due to the Brinkman term. $c$ is the complex phase velocity of compressional waves.

Use has been made of the notation

$$
\rho_{11}=(1-\varphi) \rho_{p}-\rho_{12}, \rho_{22}=\varphi \rho_{l}-\rho_{12}, \rho_{12}=-\rho_{a}
$$

By virtue of

$$
\begin{aligned}
\lambda_{1} & =\rho_{11} \rho_{22}-\rho_{12}^{2}, \lambda_{2}=-\varphi^{2} \eta / \kappa\left(\rho_{11}+\rho_{22}+2 \rho_{12}\right), \lambda_{3} \\
& =-\rho_{22} K_{p}-\left((1-\varphi)^{2} K_{l} \rho_{22} / \varphi\right)-\varphi K_{l} \rho_{11}+2(1-\varphi) K_{l} \rho_{12}, \lambda_{4} \\
& =\varphi \eta\left(K_{l}+\varphi K_{p}\right) / \kappa, \lambda_{5}=\varphi \eta\left(\rho_{11}-\rho_{22}\right), \lambda_{6}=\varphi K_{l} K_{p}, \lambda_{7} \\
& =\varphi \eta\left(-K_{p}+(2 \varphi-1 / \varphi) K_{l}\right) .
\end{aligned}
$$

Equation (15) describes the two independent solutions of Equation (11), representing the first and second waves, which are denoted by the subscripts 1 and 2 .

$$
c_{1,2}^{2}=\frac{-\left(\lambda_{3}+j \lambda_{4} \omega^{-1}+j \lambda_{5} \omega\right) \pm \sqrt{\left(\lambda_{3}+j \lambda_{4} \omega^{-1}+j \lambda_{5} \omega\right)^{2}-4\left(\lambda_{1}+j \lambda_{2} \omega^{-1}\right)\left(\lambda_{6}+j \lambda_{7} \omega\right)}}{2\left(\lambda_{1}+j \lambda_{2} \omega^{-1}\right)} .
$$


TABLE 1: Comparison of calculated and observed wave velocities.

\begin{tabular}{lcccc}
\hline Mode & Observed [15] & Observed [17] & Darcy's law & Darcy-Brinkman formulation \\
\hline First wave $(\mathrm{km} / \mathrm{s})$ & $2.27 \pm 0.06$ & $2.19 \pm 0.09$ & 2.78 & 2.36 \\
Second wave $(\mathrm{km} / \mathrm{s})$ & $0.78 \pm 0.02$ & $0.79 \pm 0.02$ & 0.67 & 0.78 \\
\hline
\end{tabular}

TABLE 2: Comparison of calculated and observed wave velocities at $780 \mathrm{kHz}$.

\begin{tabular}{lcccc}
\hline Mode & Observed [42] & Gassmann's relations & Darcy's law & Darcy-Brinkman formulation \\
\hline First wave $(\mathrm{km} / \mathrm{s})$ & $2700 \pm 55$ & 2667 & 2568 & 2362 \\
Second wave $(\mathrm{km} / \mathrm{s})$ & $1010 \pm 20$ & - & 837 & 911 \\
\hline
\end{tabular}

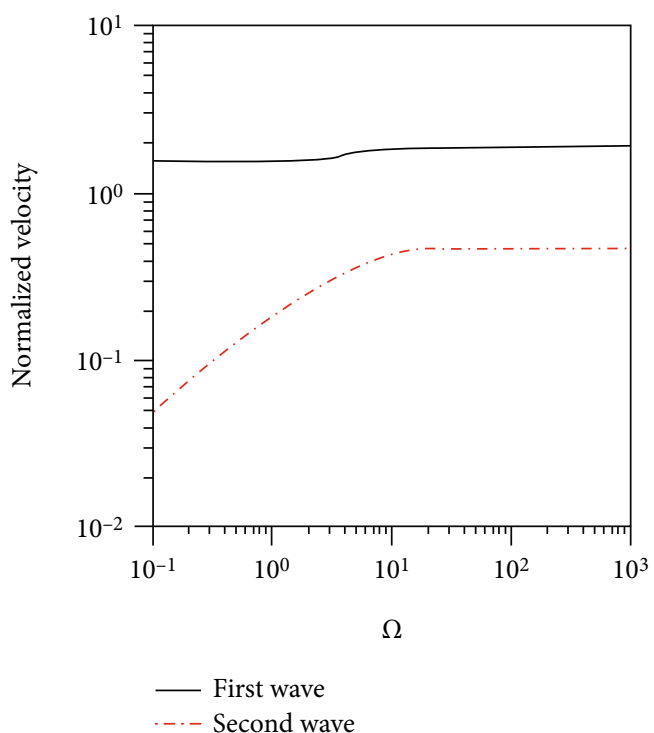

(a)

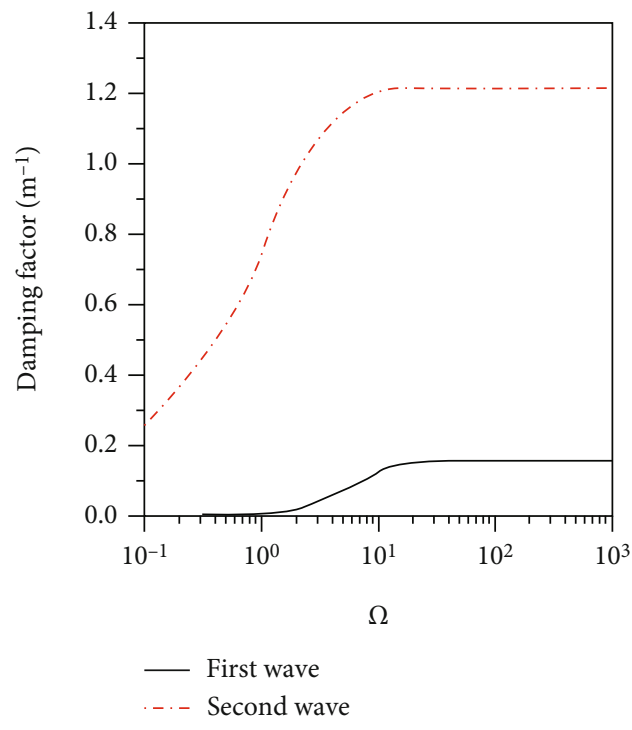

(b)

Figure 1: Velocity (a) and attenuation (b) predicted with Darcy's law for the compressional waves in water-filled sand. The velocity is normalized by the fluid velocity $1517 \mathrm{~m} / \mathrm{s}$ as a function of the normalized frequency, $\Omega=\omega / \omega_{c}$.

Let $\Phi(\omega)=\left(\lambda_{3}+j \lambda_{4} \omega^{-1}+j \lambda_{5} \omega\right)^{2}-4\left(\lambda_{1}+j \lambda_{2} \omega^{-1}\right)\left(\lambda_{6}+\right.$ $\left.j \lambda_{7} \omega\right)$. We define

$$
\begin{gathered}
\operatorname{Re} \Phi(\omega)=\lambda_{3}^{2}-\left(\lambda_{4} \omega^{-1}+\lambda_{5} \omega\right)^{2}-4 \lambda_{1} \lambda_{6}+4 \lambda_{2} \lambda_{7} \\
\operatorname{Im} \Phi(\omega)=2\left(\lambda_{3} \lambda_{4}-2 \lambda_{2} \lambda_{6}\right) \omega^{-1}+2\left(\lambda_{3} \lambda_{5}-2 \lambda_{1} \lambda_{7}\right) \omega .
\end{gathered}
$$

At a nonzero frequency, if the condition $\operatorname{Re} \Phi(\omega)=0$ is satisfied, this implies that

$\omega=\omega_{*}=\sqrt{\frac{\lambda_{3}^{2}-2 \lambda_{4} \lambda_{5}-4 \lambda_{1} \lambda_{6}+4 \lambda_{2} \lambda_{7}+\sqrt{\left(\lambda_{3}^{2}-2 \lambda_{4} \lambda_{5}-4 \lambda_{1} \lambda_{6}+4 \lambda_{2} \lambda_{7}\right)^{2}-4 \lambda_{4}^{2} \lambda_{5}^{2}}}{2 \lambda_{5}^{2}}}$.

Because $\operatorname{Im} \Phi(\omega)=0$, it has $\lambda_{1} \lambda_{4} \lambda_{7}=\lambda_{2} \lambda_{5} \lambda_{6}$. Therefore, if $\lambda_{1} \lambda_{4} \lambda_{7}=\lambda_{2} \lambda_{5} \lambda_{6}$, then at a characteristic frequency $\omega=$ $\omega_{*}$, the properties of the first and second waves are identical [25], and the biquadratic Equation (11) has a single solution, namely, $\quad c_{1}=c_{2}=\sqrt{2} / 2 \sqrt{-\lambda_{3}-j \lambda_{4} \omega^{-1}-j \lambda_{5} \omega / \lambda_{1}+j \lambda_{2} \omega^{-1}}$, which corresponds to the classical wave propagating with the mixture wave speed [15]. That is, in the saturated porous medium, a single compressional wave can propagate [25]. Note that the characteristic frequency of compressional waves represents an intrinsic property of saturated porous media which rigorously obey the criterion $\lambda_{1} \lambda_{4} \lambda_{7}=\lambda_{2} \lambda_{5} \lambda_{6}$ and only depends on the properties of the fluid and solid phases.

Supplemented with the prescribed boundary conditions of $\widehat{p}$ and $\widehat{\sigma}$ at $x=0, \widehat{p}_{0}$ and 0 , respectively, through use of Equations (7) and (8) and by superposition, the transfer functions [17] for the pore pressure and the stress waves travelling over a distance $x$ are reformulated in the form

$$
\begin{aligned}
H_{p}(x, \omega)= & \frac{\widehat{p}(x, \omega)}{\widehat{p}_{0}(\omega)}=\frac{1}{\beta_{1}-\beta_{2}}\left[\left(\beta_{1}+\frac{1-\varphi}{\varphi}\right) e^{-j \omega x / c_{1}}\right. \\
& \left.-\left(\beta_{2}+\frac{1-\varphi}{\varphi}\right) e^{-j \omega x / c_{2}}\right],
\end{aligned}
$$




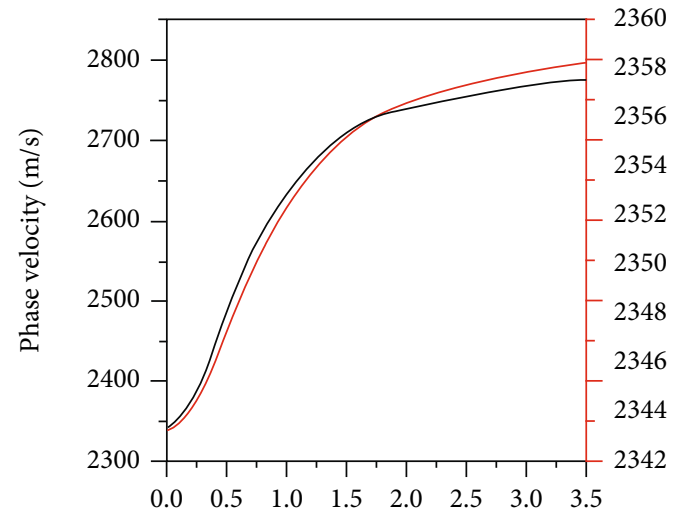

$\Omega$

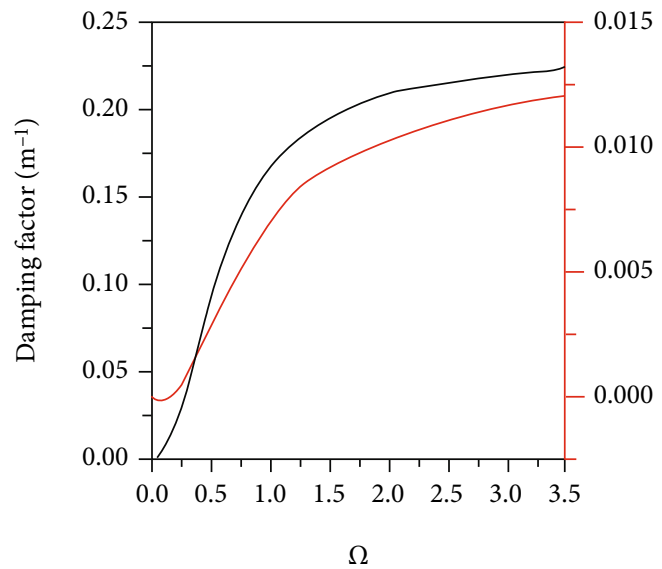

(b)

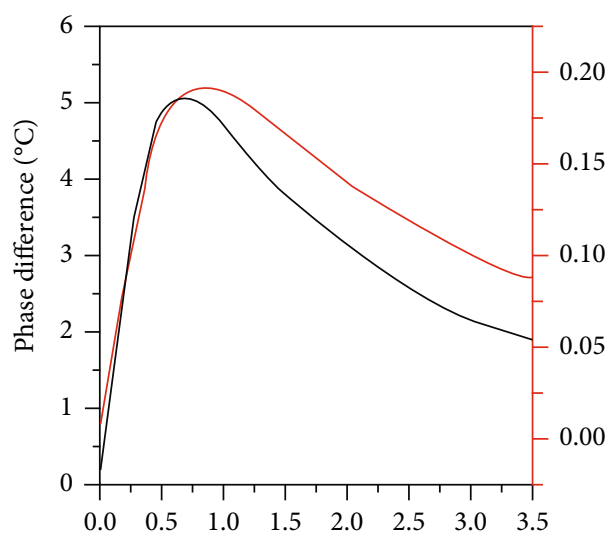

$\Omega$

(c)

Figure 2: Phase velocity (a), damping factor (b), and phase difference (c) of the first wave versus normalized frequency $\left(\Omega=\omega / \omega_{c}\right)$. The damping is the imaginary part of $-\omega / c_{i}, i=1,2$. The phase velocity is the real part of the complex wave velocity. The red line denotes the Darcy-Brinkman law and the black line Darcy's law.

$$
H_{\sigma}(x, \omega)=\frac{\widehat{\sigma}(x, \omega)}{\widehat{p}_{0}(\omega)}=\frac{K_{p}}{K_{l}} \frac{1}{\beta_{1}-\beta_{2}}\left(e^{-j \omega x / c_{1}}-e^{-j \omega x / c_{2}}\right),
$$

with

$$
\beta_{i}=\frac{\widehat{w}_{i}}{\widehat{u}_{i}}=\frac{-\rho_{12}+\left((1-\varphi) K_{l} / c_{i}^{2}\right)-\left(j \varphi^{2} \eta / k \omega\right)+\left(j \varphi \eta \omega / c_{i}^{2}\right)}{\rho_{22}-\left(\varphi K_{l} / c_{i}^{2}\right)-\left(j \varphi^{2} \eta / k \omega\right)+\left(j \varphi \eta \omega / c_{i}^{2}\right)} .
$$

The term $j \varphi \eta \omega / c_{i}^{2}$ denotes the contribution due to the Brinkman term. Equations (11), (18), and (19) constitute the fundamental equations of compressional wave fields of frequency domain in saturated porous media with the Darcy-Brinkman law included.

\section{Results and Discussion}

3.1. Model Evaluation and Validation. Experimental benchmark results $[15,17]$ from a shock tube technique are used to validate the proposed fundamental equations of compressional wave fields of frequency domain with the DarcyBrinkman law included. In the experimental setup, a vertical shock tube is utilized to trigger a step pressure loading at the top of a porous cylinder located in the test section of the tube. By measuring simultaneously pore pressures and strains at observation points, the in-phase and out-ofphase wave modes and the wave speeds are observed. The following mechanical properties [17] of a sandstone saturated with water are utilized: $\varphi=0.3, \rho_{l}=990 \mathrm{~kg} / \mathrm{m}^{3}, \rho_{p}=$ $2650 \mathrm{~kg} / \mathrm{m}^{3}, \eta=1.0 \times 10^{-3} \mathrm{kgm}^{-1} \mathrm{~s}^{-1}, K_{l}=2.2 \mathrm{GPa}, K_{p}=4.5$ $\mathrm{GPa}, \kappa=5.6 \times 10^{-11} \mathrm{~m}^{2}$, and $\alpha_{\infty}=1.45$. Since the constrained modulus of the solid and the added mass are certainly the important effects, it has been verified from the experiments $[15,17]$ that the effective constrained modulus of the solid has a value of $4.5 \mathrm{GPa}$, and the effective added mass parameter is 1.45. Quantitative information on compressional wave speeds is compared with theoretical predictions, as shown in Table 1.

The calculated wave speeds from the Darcy-Brinkman law are well agreement with the observed wave speeds. The 


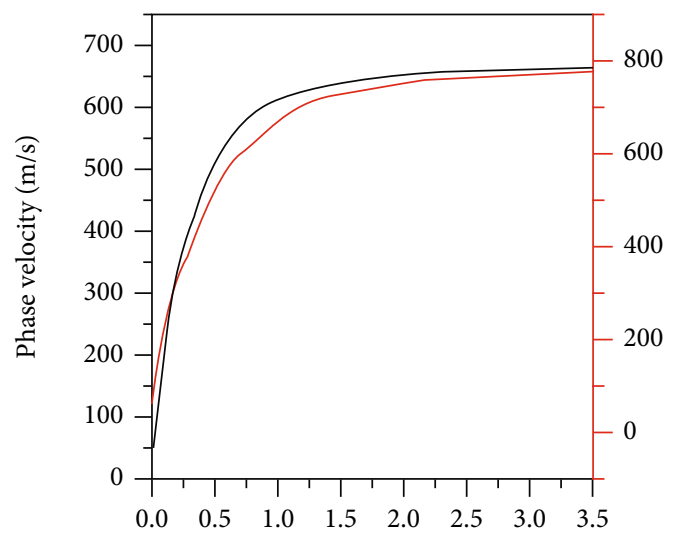

$\Omega$

(a)

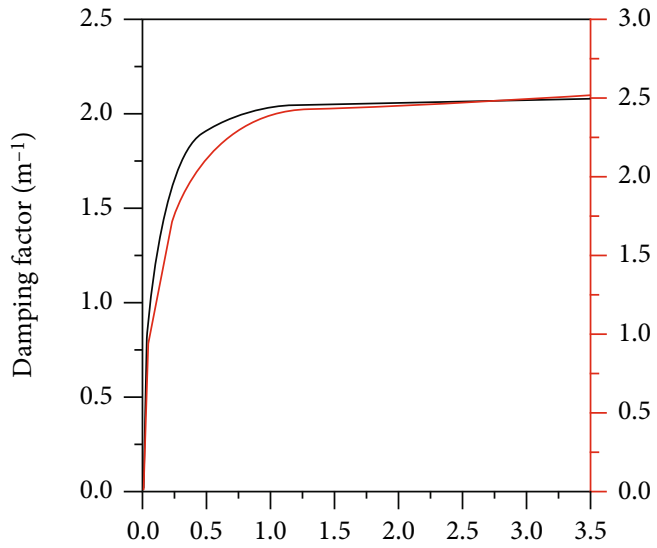

$\Omega$

(b)

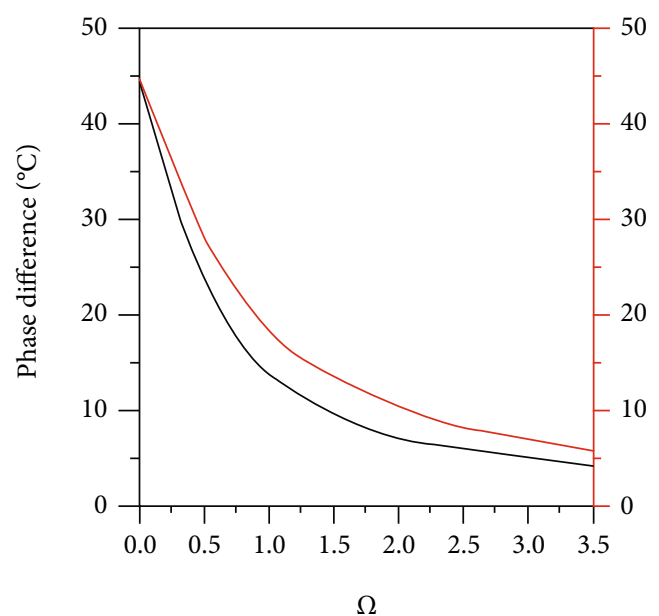

(c)

Figure 3: Phase velocity (a), damping factor (b), and phase difference (c) of the second wave versus normalized frequency $\left(\Omega=\omega / \omega_{c}\right)$. The red line presents the Darcy-Brinkman law and the black line Darcy's law.

introduction of the Brinkman term clearly improves the description of the damping for the second compressional wave [17]. The primary emphasis here is that the fluid density and porosity are rigorously dependent of pore pressure and effective stress by the constitutive Equations (18), that the intrinsic density is a constant, and that the compression or expansion of the solid can be fully described in terms of the porosity.

Bouzidi and Schmitt [42] measured the speed of the compressional waves in a water-loaded, porous sintered glass bead plate with a novel pair of ultrasonic transducers consisting of a large transmitter and a near-point receiver. Updated observations of the two compressional wave modes are obtained. The following mechanical properties [42] are utilized: $\varphi=0.391, \rho_{l}=995 \mathrm{~kg} / \mathrm{m}^{3}, \rho_{p}=2445 \mathrm{~kg} / \mathrm{m}^{3}, \eta=1.0$ $\times 10^{-3} \mathrm{kgm}^{-1} \mathrm{~s}^{-1}, K_{l}=2.209 \mathrm{GPa}, K_{p}=4.826 \mathrm{GPa}, \kappa=1.99$ $\times 10^{-11} \mathrm{~m}^{2}$ and $\alpha_{\infty}=1.44$. The velocities are calculated from Equation (11) given in Table 2.

Compared to the experimental results, Darcy's law and the Darcy-Brinkman formulation underestimate the veloci- ties of the compressional waves. One key factor included in the dispersion relation (Equation (11)) is the effective constrained modulus of the solid $K_{p}$ that is not available in the reference [42]. In the calculation, the frame bulk modulus is used. As stated by Bouzidi and Schmitt [42], other factors such as heterogeneity of the porosity might partially lead to this discrepancy. The key fact is that the fluid density and porosity are rigorously related to pore pressure and effective stress by the constitutive equations and that the deformation of the solid can be fully described in terms of the porosity. In the experiments, the emitted ultrasonic waves cannot cause the change of porosity.

Albert [16] simulated wave propagation in a watersaturated porous material. Here, we demonstrate that in the limit where the Brinkman term vanishes, the result for Darcy's law is recovered. Formation and saturated parameters used are those as reported by Albert [16]. Figure 1 shows that the velocity and attenuation dispersion curves obtained using our model show significant dispersion of the same order as that predicted by the original Biot theory [16]. 


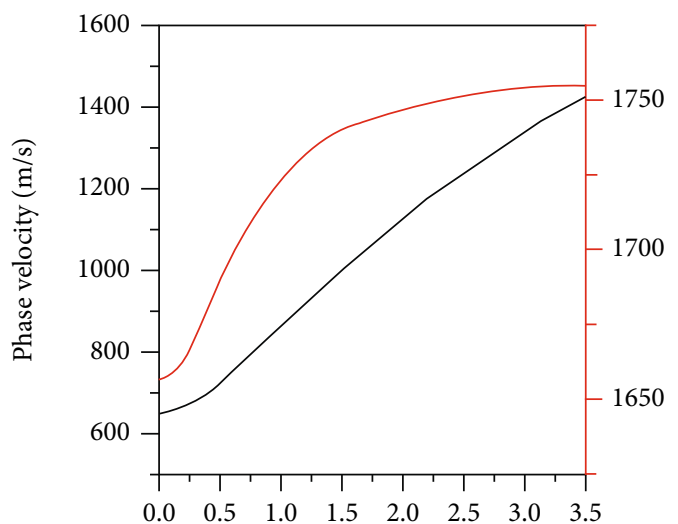

$\Omega$

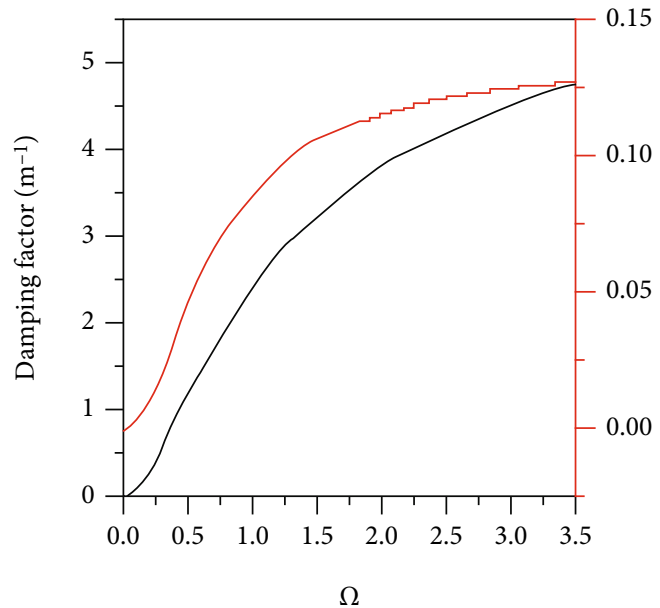

(b)

(a)

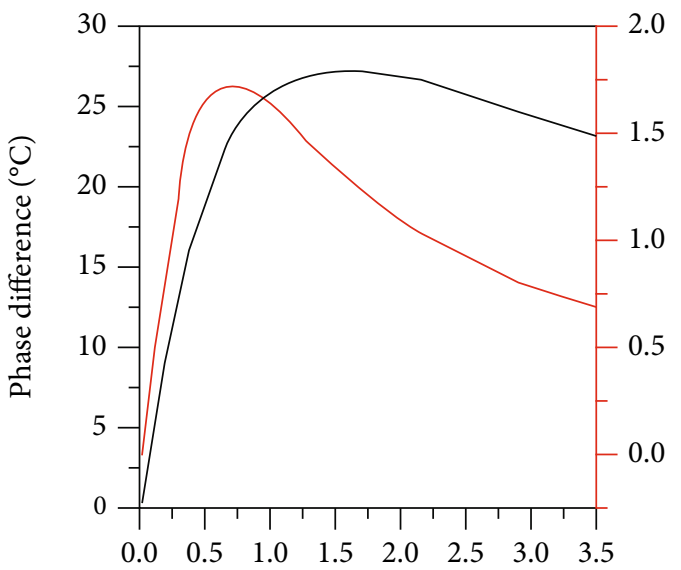

$\Omega$

(c)

Figure 4: Phase velocity (a), damping factor (b), and phase difference (c) of the wave versus normalized frequency $\left(\Omega=\omega / \omega_{c}\right)$ under the condition of $\lambda_{1} \lambda_{4} \lambda_{7}=\lambda_{2} \lambda_{5} \lambda_{6}$. The red line presents the Darcy-Brinkman law and the black line Darcy's law.

3.2. Phase Velocity and Damping of the First and Second Kind of Waves. The following mechanical properties [17] of a sandstone saturated with water are used: $\varphi=0.3, \rho_{l}=$ $990 \mathrm{~kg} / \mathrm{m}^{3}, \rho_{p}=2650 \mathrm{~kg} / \mathrm{m}^{3}, \eta=1.0 \times 10^{-3} \mathrm{kgm}^{-1} \mathrm{~s}^{-1}, K_{l}=$ $2.2 \mathrm{GPa}, K_{p}=4.5 \mathrm{GPa}, \kappa=5.6 \times 10^{-11} \mathrm{~m}^{2}$, and $\alpha_{\infty}=1.45$. Figures 1-3 illustrate the normalized frequency-dependent $\left(\Omega=\omega / \omega_{c}\right)$ dispersion profiles of the phase velocity, damping, and phase lag. As expected, the first wave (see Figure 2) is a slightly decaying high-velocity mode, and the relative motion of fluid and solid phases is the in-phase $[26,32,33]$, exhibiting a pressure-relaxation phenomenon [30], whereas the second wave (see Figure 3) is a strongly damping slow-velocity mode and the movement of fluid and solid phases is the out-of-phase [32, 33], showing the feature of diffusion waves [28], in which the pore pressure predominates over the effective stress [26]. As seen in Figure 4 , under the condition of $\lambda_{1} \lambda_{4} \lambda_{7}=\lambda_{2} \lambda_{5} \lambda_{6}$, the properties of the first and second waves coincide. The two wave fronts emerge into a single front [16], and the saturated porous medium behaves a single continuum exhibiting internal dissipation. Under a certain relationship $\lambda_{1} \lambda_{4} \lambda_{7}=$ $\lambda_{2} \lambda_{5} \lambda_{6}$ between the fluid and solid parameters in which only exists a single wave, the phase velocity is underestimated, while the damping factor and phase lag are overestimated by Darcy's law. Compared to the Darcy-Brinkman law, Darcy's law overestimates the phase velocity, damping factor, and phase lag of the first wave, while underestimates the phase velocity, damping factor, and phase difference of the second wave. The Darcy-Brinkman law does not lead to the change of the in-phase motion into out-of-phase motion in the Frenkel-Biot wave modes [26], as can be seen that the second wave is of opposite polarization compared to the first compression wave $[32,33]$, which is consistent with the Gassmann equation [43]. The effects of the internal friction arising from viscous shear stress $[8,36,37]$ on propagation of compressional waves are of significant importance.

3.3. Wave Fields of Pore Pressure and Stress. Propagation of pore pressure and stress waves is analytically investigated in a one-dimensional semi-infinite domain subject to the 

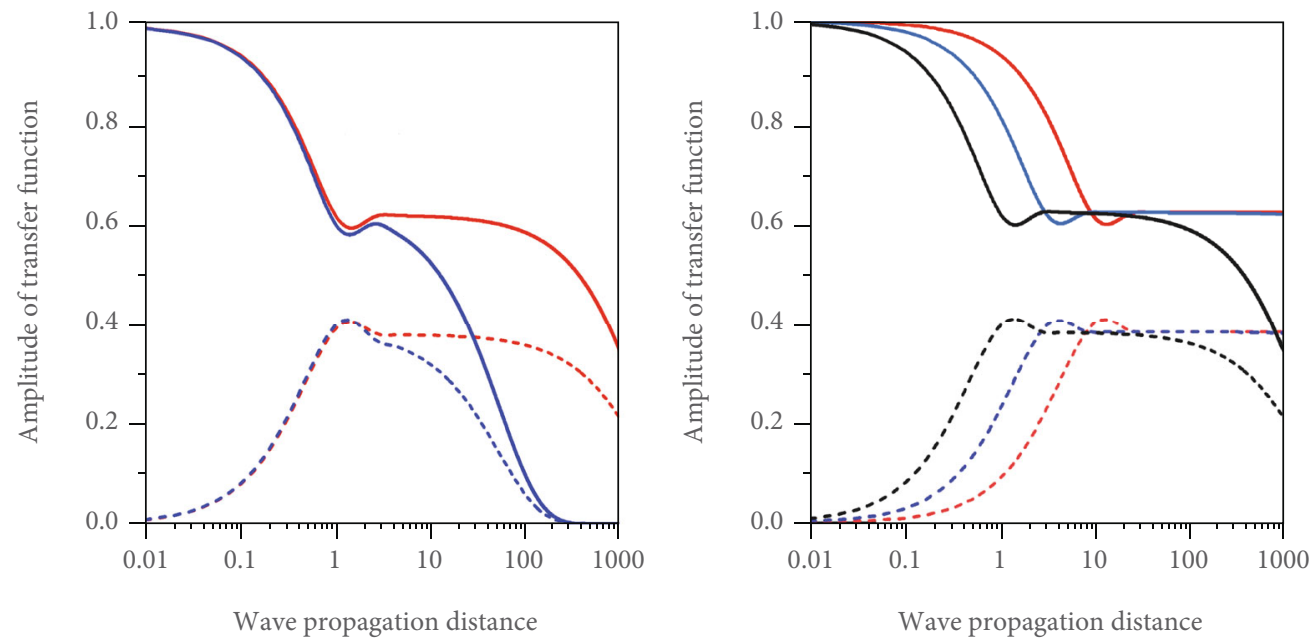

$$
\begin{aligned}
& H_{p}(x, \omega) \\
& - \text { Darcy-brinkman } \\
& - \text { Darcy } \\
& H_{\sigma}(x, \omega) \\
& \text { - - Darcy-brinkman } \\
& \text { - - - Darcy }
\end{aligned}
$$

$$
\begin{array}{ll}
H_{p}(x, \omega) & H_{\sigma}(x, \omega) \\
-10 \mathrm{HZ} & ---10 \mathrm{HZ} \\
-100 \mathrm{HZ} & ---100 \mathrm{HZ} \\
-1000 \mathrm{HZ} & ---1000 \mathrm{HZ}
\end{array}
$$

(b)

Figure 5: Normalized amplitude of pore pressure and stress wave versus travelling distance, (a) comparison of Darcy's law with the DarcyBrinkman law for an angular frequency of $1000 \mathrm{~Hz}$, and (b) based on the Darcy-Brinkman effect with angular frequency as a parameter for $\eta=1.0 \times 10^{-3} \mathrm{kgm}^{-1} \mathrm{~s}^{-1}$. The solid line denotes the transfer function of the pore pressure, and the dash-dot line represents the transfer function of the stress. The wave travelling distance is normalized by the viscous penetration length $\delta=\sqrt{2 \mu / \omega \rho_{l}}$ at frequency of $2 \times 10^{-6} \mathrm{~Hz}$.

step loading, $\Delta p$ of pore pressure at $t=0, x=0$. Figure 4 shows a simulation of the frequency dependence of the amplitude of the pore pressure and the stresses over a wave travelling distance. From the comparison of Darcy's law with the Darcy-Brinkman law, it is found that the Brinkman term is negligible in the near field, while the viscous shear stress significantly influences the wave motion in the far field (see Figure 5(a)). Figure 5(b) shows the interference pattern of the wave fronts. A key result is that during the wave motion, the amplitude of the pore pressure generally decreases, compared to the shear-induced pore pressure changes [44]. The magnitude of the stress firstly increases and then decreases back to its initial value. The amplitudecurve crossovers occur as the interplay between greater phase lags due to increasing frequency [45]. The two-wave modes [43, 46], dissipative and diffusive patterns [47], are demonstrated clearly.

Based on the Darcy-Brinkman law, Figure 6(a) shows the simulation of the pore pressure and stress waves for various viscosities, $\eta$. The viscous dissipation of energy [35, 37] considerably influences the propagation of the pore pressure and stress waves. The pore pressure and stress waves exhibit dissipative and dispersive behaviors [24, 46-48]. The amplitude-curve crossovers are due to the spatial superposition of the confined waves acting like interference patterns $[45,49]$. As can be seen in Figure 6(b), the amplitudes of pore pressure and stress are insensitive to the value of the added-mass coefficient. The regime where the pore pressure (or stress) curves coincide implies that the pore pressure (or stress) is in that case completely independent of $\alpha_{\infty}$ and corresponds to the situation $\widehat{u}=\widehat{w}$ as shown from the field Equations (5) and (6). The pressure-coherent stress follows a single gamma distribution, compared to the flowinduced stress in saturated porous media [50].

3.4. Discussions of the Darcy-Brinkman-Biot Formulation. It is noted that the assumption of Darcy's law is not valid if the frequency exceeds a Biot's critical frequency [14] above which the compressional wave attenuation is strongly controlled by the viscous shear stress, and the DarcyBrinkman law should be considered to address the effects of non-Darcy flow on compressional wave motions. Moreover, for compressional elastic wave propagation in Newtonian fluid-saturated porous media, as pointed by Carrillo and Bourg [37], the Darcy term is not adapted to describe systems with both macropores and micropores, which contain two characteristic length scales. Fluid flow in two-scale porous media is governed by a general coupled system of equations, which approximates the Navier-Stokes equations in fluid-filled macropores and resembles the equations for poroelasticity in microporous regions.

Now, it has to be realized that in the theoretical analysis, we have assumed that the nonlinear inertial terms in the momentum equations of the fluid and solid can be neglected for the frequency below the critical Biot frequency. This assumption is also reasonable to a small Reynolds number 


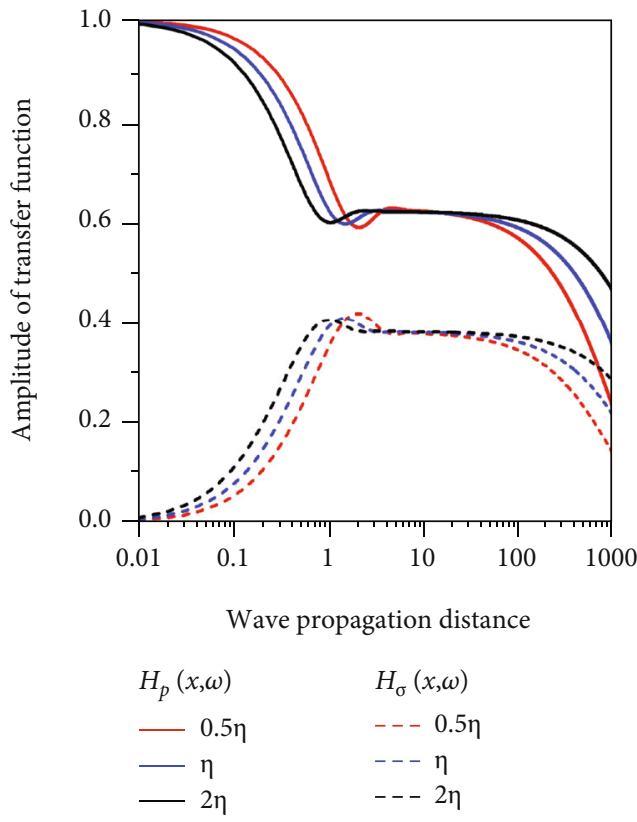

(a)

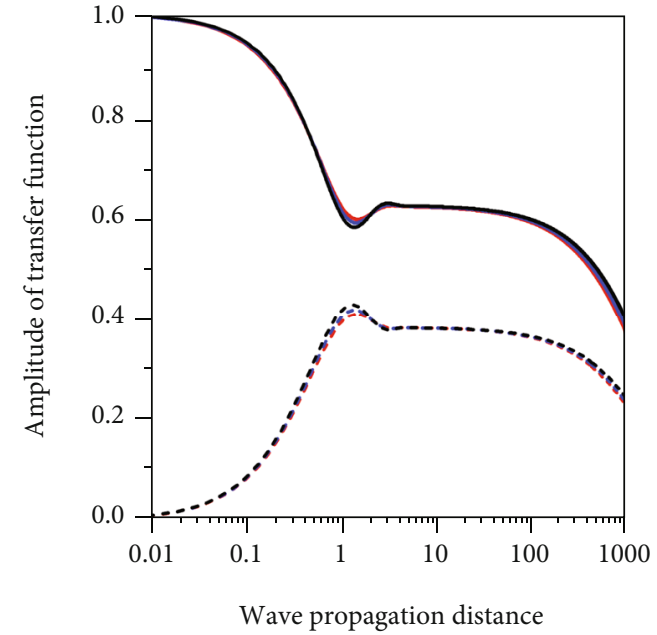

$\begin{array}{ll}H_{p}(x, \omega) & H_{\sigma}(x, \omega) \\ -a_{\infty=1.45} & ---a_{\infty=1.45} \\ -a_{\infty=2.05} & ---a_{\infty}=2.05 \\ -a_{\infty}=2.7 & ---a_{\infty}=2.7\end{array}$

(b)

Figure 6: Normalized amplitude of pore pressure and stress versus wave travelling distance based on Darcy-Brinkman law with $\eta$ as a parameter (a) and with added-mass coefficient $\alpha_{\infty}$ as a parameter (b) at an angular frequency of $1000 \mathrm{~Hz}$. The solid line denotes the transfer function of the pore pressure, and the dash-dot line represents the transfer function of the stress. The wave travelling distance is normalized by the viscous penetration length $\delta=\sqrt{2 \mu / \omega \rho_{l}}$ at frequency of $2 \times 10^{-6} \mathrm{~Hz}$.

$(\operatorname{Re}<1)$ within the microporous region [37]. In the limit of high frequencies, the frequency-dependent interaction forces between solid and fluid can be described in terms of the high-frequency added mass $j \omega \rho_{a}$. However, the DarcyBrinkman-Biot formulation [37] does not include the Forchheimer equation.

Future work involves the addition of Forchheimer term $[51,52]$, solid dissipation, and viscoelasticity and relaxation effects to the momentum conservation laws of compressional waves in saturated porous media. Of particular interest is that previous mathematical approaches $[52,53]$ of the Laplace transform, Fourier transform, and matrix transfer would be extended by solving the one-dimensional DarcyBrinkman-Biot formulation of frequency domain.

\section{Conclusions}

As a conclusion, we show that the propagation of the pore pressure and stress waves in water-saturated elastic porous media with the Darcy-Brinkman law involved. The viscous shear stress significantly influences the phase velocity, damping, and phase lag of the first and second waves. The Darcy-Brinkman law does not lead to the change of the inphase motion into out-of-phase motion in the Frenkel-Biot wave modes. The second wave is of opposite polarization compared to the first wave. The pore pressure-coherent stress follows a single gamma distribution.

Compared to the Darcy-Brinkman law, Darcy's law overestimates the wave velocity, damping, and phase lag of the first wave, while underestimates the wave velocity, damping, and phase difference of the second wave. The aver- age error overestimated by the Darcy law is $317.01 \mathrm{~m} / \mathrm{s}$ for the phase velocity, $0.17 \mathrm{~m}^{-1}$ for the damping, and 3.05 (degree) for the phase lag with respect to the first wave. The average error underestimated due to the Darcy law is $79.15 \mathrm{~m} / \mathrm{s}$ for the phase velocity, $0.36 \mathrm{~m}^{-1}$ for the damping and 3.05 (degree) for the phase lag with respect to the second wave. A characteristic frequency is found to be a decisive condition for the coincidence of the properties of Biot waves of the first and second kinds. The transfer functions of the pore pressure and stress waves are reformulated. The DarcyBrinkman law describes the dissipative and dispersive behavior of compressional wave propagation in a water-saturated elastic porous medium. The introduction of the Brinkman term yields a better understanding of the Frenkel-Biot waves in saturated porous media. For compressional elastic wave propagation in Newtonian fluid-saturated porous media, when the error of Darcy law is large enough, non-Darcy effects should be considered using the Darcy-Brinkman law.

\section{Appendix}

As stated by the reference [14], the momentum conservation laws for the fluid and the solid phases are expressed as

$$
\begin{gathered}
\frac{\partial}{\partial t}\left(\rho_{22} w+\rho_{12} u\right)=-\varphi \frac{\partial p}{\partial x}+\frac{\varphi^{2} \eta}{k}(u-w) \\
\frac{\partial}{\partial t}\left(\rho_{11} u+\rho_{12} w\right)=-\frac{\partial \sigma}{\partial x}-(1-\varphi) \frac{\partial p}{\partial x}-\frac{\varphi^{2} \eta}{k}(u-w) .
\end{gathered}
$$


The second-order term, called the Brinkman term $\varphi \eta$ $\left(\partial^{2} / \partial x^{2}\right)(u-w)$ recovers the viscous shear stress. By introducing the Brinkman term to the momentum conservation equations ((21) and (22)), it yields

$$
\frac{\partial}{\partial t}\left(\rho_{22} w+\rho_{12} u\right)=-\varphi \frac{\partial p}{\partial x}+\frac{\varphi^{2} \eta}{k}(u-w)+\varphi \eta \frac{\partial^{2}}{\partial x^{2}}(u-w)
$$

$\frac{\partial}{\partial t}\left(\rho_{11} u+\rho_{12} w\right)=-\frac{\partial \sigma}{\partial x}-(1-\varphi) \frac{\partial p}{\partial x}-\frac{\varphi^{2} \eta}{k}(u-w)-\varphi \eta \frac{\partial^{2}}{\partial x^{2}}(u-w)$.

Let all quantities that are sinusoidal functions [17, 37] of time contain a factor $e^{j \omega t}$, we obtain

$$
j \omega \varphi \rho_{l} \widehat{w}=-\varphi \frac{\partial \widehat{p}}{\partial x}+\varphi \eta \frac{\partial^{2}(\widehat{u}-\widehat{w})}{\partial x^{2}}+\left(j \omega \rho_{a}+\frac{\varphi^{2} \eta}{\kappa}\right)(\widehat{u}-\widehat{w}),
$$

$j \omega(1-\varphi) \rho_{p} \widehat{u}=-\frac{\partial \widehat{\sigma}}{\partial x}-(1-\varphi) \frac{\partial \widehat{p}}{\partial x}-\varphi \eta \frac{\partial^{2}(\widehat{u}-\widehat{w})}{\partial x^{2}}-\left(j \omega \rho_{a}+\frac{\varphi^{2} \eta}{\kappa}\right)(\widehat{u}-\widehat{w})$.

Caret $(\wedge)$ represents the complex amplitude, where $j^{2}=-1$.

\section{Nomenclature}

$\widehat{p}$ : $\quad$ Pore pressure

$\widehat{\sigma}: \quad$ Effective stress

$\widehat{u}$ : Actual velocities of the fluid

$\widehat{w}$ : Actual velocities of the solid

$c$ : Complex phase velocity of compressional waves

$\varphi$ : Porosity

$\kappa: \quad$ Frequency-independent permeability

$\omega$ : Angular frequency

$\omega_{c}:$ Critical Biot frequency

$\omega_{*}$ : Characteristic frequency

$K_{l}$ : Bulk modulus of the fluid

$K_{p}$ : Constrained modulus of the solid

$\alpha_{\infty}$ : Added mass coefficient

$\delta: \quad$ Viscous penetration length

$\eta$ : $\quad$ Fluid viscosity coefficient

$x$ : Distance

$t$ : Time.

\section{Data Availability}

No new data were created or analyzed in this study. Data sharing is not applicable to this article.

\section{Conflicts of Interest}

The authors declare that they have no conflict of interest.

\section{Acknowledgments}

The authors are sincerely grateful to the anonymous reviewers for their constructive comments that have led to significant revision and clarification of the original manuscript. This work was funded by the National Key R\&D Program of China (2018YFC1503403), the National Natural Science Foundation of China (grant no. 41874113), and research grants from the National Institute of Natural Hazards, Ministry of Emergency Management of China (grant no. ZDJ2019-15).

\section{References}

[1] D. O. Riese and G. H. Wegdam, "Sound propagation in suspensions of colloidal spheres with viscous coupling," Physical Review Letters, vol. 82, no. 8, pp. 1676-1679, 1999.

[2] F. Ebrahimi, S. H. S. Hosseini, and A. Singhal, "A comprehensive review on the modeling of smart piezoelectric nanostructures," Structural Engineering and Mechanics, vol. 74, no. 5, pp. 611-633, 2020.

[3] F. Ebrahimi, M. Karimiasl, and A. Singhal, "Magneto-electroelastic analysis of piezoelectric-flexoelectric nanobeams rested on silica aerogel foundation," Engineering with Computers, vol. 37, no. 2, pp. 1007-1014, 2021.

[4] S. Chaudhary, S. A. Sahu, and A. Singhal, "On secular equation of $\mathrm{SH}$ waves propagating in pre-stressed and rotating piezocomposite structure with imperfect interface," Journal of Intelligent Material Systems and Structures, vol. 29, no. 10, pp. 2223-2235, 2018.

[5] S. Abhinav, S. Sanjeev, C. Soniya, and B. Juhi, "Initial and couple stress influence on the surface waves transmission in material layers with imperfect interface," Materials Research Express, vol. 6, no. 10, 2019.

[6] A. Singhala and S. A. Sahu, "Transference of Rayleigh waves in corrugated orthotropic layer over a pre-stressed orthotropic half-space with self-weight," Procedia Engineering, vol. 173, pp. 972-979, 2017.

[7] S. Chaudhary, S. A. Sahu, A. Singhal, and S. Nirwal, "Interfacial imperfection study in pre-stressed rotating multiferroic cylindrical tube with wave vibration analytical approach," Materials Research Express, vol. 6, no. 10, 2019.

[8] S. A. Sahu, A. Singhal, and S. Chaudhary, "Surface wave propagation in functionally graded piezoelectric material: an analytical solution," Journal of Intelligent Material Systems and Structures, vol. 29, no. 3, pp. 423-437, 2018.

[9] A. Singhal, S. A. Sahu, and S. Chaudhary, "Approximation of surface wave frequency in piezo-composite structure," Composites Part B: Engineering, vol. 144, pp. 19-28, 2018.

[10] M. K. Singh, S. A. Sahu, A. Singhal, and S. Chaudhary, "Approximation of surface wave velocity in smart composite structure using Wentzel-Kramers-Brillouin method," Journal of Intelligent Material Systems and Structures, vol. 29, no. 18, pp. 3582-3597, 2018.

[11] S. Chaudhary, S. A. Sahu, N. Dewangan, and A. Singhal, "Stresses produced due to moving load in a prestressed piezoelectric substrate," Mechanics of Advanced Materials and Structures, vol. 26, no. 12, pp. 1028-1041, 2019.

[12] A. Singhal, S. A. Sahu, S. Nirwal, and S. Chaudhary, "Anatomy of flexoelectricity in micro plates with dielectrically highly/ 
weakly and mechanically complaint interface," Materials Research Express, vol. 6, no. 10, 2019.

[13] Y. Frenkel, "On the theory of seismic and seismoelectric phenomena in moist soil," Journal of Physics, vol. 3, no. 5, pp. 230-241, 1944.

[14] M. A. Biot, "Theory of propagation of elastic waves in a fluidsaturated porous solid. I. Low-frequency range," The Journal of the Acoustical Society of America, vol. 28, no. 2, pp. 168-178, 1956.

[15] J. G. M. van der Grinten, M. E. H. van Dongen, and H. van der Kogel, "A shock-tube technique for studying pore-pressure propagation in a dry and water-saturated porous medium," Journal of Applied Physics, vol. 58, no. 8, pp. 2937-2942, 1985.

[16] D. G. Albert, "A comparison between wave propagation in water-saturated and air-saturated porous materials," Journal of Applied Physics, vol. 73, no. 1, pp. 28-36, 1993.

[17] J. G. M. van der Grinten and E. H. Marinus, "Strain and pore pressure propagation in a water-saturated porous medium," Journal of Applied Physics, vol. 62, no. 12, pp. 4682-4687, 1987.

[18] M. S. Diallo and E. Appel, "Acoustic wave propagation in saturated porous media: reformulation of the Biot/Squirt flow theory," Journal of Applied Geophysics, vol. 44, no. 4, pp. 313-325, 2000.

[19] F. Gimalitdinov, "Rates of propagation and decay of weak discontinuities of stress and pressure in a saturated, arbitrarily cemented porous medium," Fluid Dynamics, vol. 3, no. 6, pp. 119-120, 1972.

[20] D. H. Yang and Z. J. Zhang, "Poroelastic wave equation including the Biot/squirt mechanism and the solid/fluid coupling anisotropy," Wave Motion, vol. 35, no. 3, pp. 223-245, 2002.

[21] P. B. Nagy, L. Adler, and B. P. Bonner, "Slow wave propagation in air-filled porous materials and natural rocks," Applied Physics Letters, vol. 56, no. 25, pp. 2504-2506, 1990.

[22] R. F. Ganiev, S. A. Petrov, and L. E. Ukrainskii, "Nonlinear wave effects in a fluid-saturated porous medium," Fluid Dynamics, vol. 27, no. 1, pp. 55-59, 1992.

[23] I. Y. Edelman, "Propagation of nonlinear waves in a porous medium with two-phase saturation by a liquid and a gas," Fluid Dynamics, vol. 31, no. 4, pp. 552-559, 1996.

[24] D. M. J. Smeulders and M. E. H. van Dongen, "Wave propagation in porous media containing a dilute gas-liquid mixture: theory and experiments," Journal of Fluid Mechanics, vol. 343, pp. 351-373, 1997.

[25] D. N. Mikhailov, "Difference between the longitudinal Frenkel-Biot waves in water-and gas-saturated porous media," Fluid Dynamics, vol. 41, no. 1, pp. 112-120, 2006.

[26] S. Z. Dunin, D. Mikhailov, and V. N. Nikolayevskii, "Longitudinal waves in partially saturated porous media: the effect of gas bubbles," Journal of Applied Mathematics and Mechanics, vol. 70, no. 2, pp. 251-263, 2006.

[27] M. Atkinson and K. Kytomaa, "Acoustic wave speed and attenuation in suspensions," International Journal of Multiphase Flow, vol. 18, no. 4, pp. 577-592, 1992.

[28] D. X. Yang, M. Doschoris, and L. Z. Zhang, "Analysis of pressure diffusion-wave fields in matrix-fracture media using Green functions of frequency domain," Journal of Applied Physics, vol. 124, no. 7, 2018.

[29] S. Minato and R. Ghose, "Low-frequency guided waves in a fluid-filled borehole: simultaneous effects of generation and scattering due to multiple fractures," Journal of Applied Physics, vol. 121, no. 10, 2017.
[30] S. Diego, M. Paradelo, A. Corral, and J. E. L. Periago, "Pressure jumps during drainage in macroporous soils," Vadose Zone Journal, vol. 16, no. 13, pp. 1-12, 2017.

[31] L. De Ryck, J. P. Groby, P. Leclaire et al., “Acoustic wave propagation in a macroscopically inhomogeneous porous medium saturated by a fluid," Applied Physics Letters, vol. 90, no. 18, 2007.

[32] Z. W. Cui, J. X. Liu, and K. X. Wang, "Elastic waves in nonNewtonian (Maxwell) fluid-saturated porous media," Waves in Random Media, vol. 13, no. 3, pp. 191-203, 2003.

[33] D. Tsiklauri and I. Beresnev, "Properties of elastic waves in a non-Newtonian (Maxwell) fluid-saturated porous medium," Transport in Porous Media, vol. 53, no. 1, pp. 39-50, 2003.

[34] G. Q. Li, P. F. Zhang, and J. S. Sun, "A new model describing the interaction between fluid pressure wave in pores and $\mathrm{P}$ wave in rock matrix," Geophysics, vol. 82, no. 4, pp. MR105MR109, 2017.

[35] F. J. Valdes-Parada, J. A. Ochoa-Tapia, and J. AlvarezRamirez, "On the effective viscosity for the Darcy-Brinkman equation," Physica A: Statistical Mechanics and its Applications, vol. 385, no. 1, pp. 69-79, 2007.

[36] T. Phatak and K. B. Nakshatrala, "Effect of viscous shearing stresses on optimal material designs for flow of fluids through porous media," Physics of Fluids, vol. 33, no. 6, 2021.

[37] F. J. Carrillo and I. C. Bourg, "A Darcy-Brinkman-Biot approach to modeling the hydrology and mechanics of porous media containing macropores and deformable microporous regions," Water Resources Research, vol. 55, no. 10, pp. 80968121, 2019.

[38] O. Manor, “Acoustic flow in porous media," Journal of Fluid Mechanics, vol. 920, 2021.

[39] S. Whitaker, "The Forchheimer equation: a theoretical development," Transport in Porous Media, vol. 25, no. 1, pp. $27-$ 61, 1996.

[40] D. X. Yang, Z. Q. Xue, and S. A. Mathias, “Analysis of momentum transfer in a lid-driven cavity containing a BrinkmanForchheimer medium," Transport in Porous Media, vol. 92, no. 1, pp. 101-118, 2012.

[41] A. Levy, G. Ben-dor, and S. Sorek, "Numerical investigation of the propagation of shock waves in rigid porous materials: development of the computer code and comparison with experimental results," Journal of Fluid Mechanics, vol. 324, pp. 163-179, 1996.

[42] Y. Bouzidi and D. R. Schmitt, "Measurement of the speed and attenuation of the Biot slow wave using a large ultrasonic transmitter," Journal of Geophysical Research, vol. 114, no. B8, p. B08201, 2009.

[43] B. Gurevich, "Comparison of the low-frequency predicitons of Biot's and de Boer's poroelasticity theories with Gassmann's equation," Applied Physics Letters, vol. 91, no. 9, 2007.

[44] M. A. Koenders and N. Petford, "Shear-induced pressure changes and seepage phenomena in a deforming porous layer-III," Geophysical Journal International, vol. 171, no. 2, pp. 943-953, 2007.

[45] Y. Wang, S. Dong, and Y. Luo, "Model-based interferometric interpolation method," Geophysics, vol. 75, no. 6, p. WB211, 2010.

[46] V. Kazemi-Kamyab, K. Subramaniam, and Y. Andreopoulos, "Stress transmission in porous materials impacted by shock waves," Journal of Applied Physics, vol. 109, no. 1, 2011. 
[47] D. Kalisman, S. Sorek, A. Yakirevich, and T. Kamai, "A macroscopic analytical model for pressure wave propagation in the water of a variably saturated porous medium," Vadose Zone Journal, vol. 18, no. 1, 2019.

[48] J. Aichele, B. Giammarinaro, M. Reinwald, G. Le Moign, and S. Catheline, "Capturing the shear and secondary compression waves: high-frame-rate ultrasound imaging in saturated foams," Physical Review Letters, vol. 123, no. 14, 2019.

[49] D. A. Gubaidullin and Y. V. Fedorov, "Acoustic wave incidence on a multilayer medium containing a bubbly fluid layer," Fluid Dynamics, vol. 52, no. 1, pp. 107-114, 2017.

[50] R. S. Voronov, S. B. VanGordon, V. I. Sikavitsas, and D. V. Papavassiliou, "Distribution of flow-induced stresses in highly porous media," Applied Physics Letters, vol. 97, no. 2, 2010.

[51] E. Abobaker, A. Elsanoose, F. Khan, M. A. Rahman, A. Aborig, and S. Butt, "Comparison of crushed-zone skin factor for cased and perforated wells calculated with and without including a tip-crushed zone effect," Geofluids, vol. 2020, Article ID 3689964, 13 pages, 2020.

[52] S. A. Mathias, J. N. McElwaine, and J. G. Gluyas, "Heat transport and pressure buildup during carbon dioxide injection into depleted gas reservoirs," Journal of Fluid Mechanics, vol. 756, pp. 89-109, 2014

[53] S. V. Kirilovskiy, A. A. Maslov, S. G. Mironov, and T. V. Poplavskaya, "Application of the skeleton model of a highly porous cellular material in modeling supersonic flow past a cylinder with a forward gas-permeable insert," Fluid Dynamics, vol. 53, no. 3, pp. 409-416, 2018. 